\title{
Preparation and physicochemical characterisation of functionalised multi-walled carbon nanotubes
}

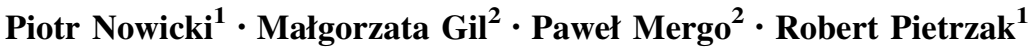

Received: 9 October 2015/Revised: 2 November 2015/Accepted: 11 November 2015/Published online: 17 November 2015

(C) The Author(s) 2015. This article is published with open access at Springerlink.com

\begin{abstract}
A series of new functionalised carbonaceous materials were prepared by means of oxidation and ammoxidation of commercially available multi-walled carbon nanotubes. The effect of oxygen and nitrogen doping on the textural, surface as well as thermal properties of the prepared adsorbents was tested. The materials were characterized by elemental analysis, low-temperature nitrogen sorption, determination of the surface oxygen groups content as well as by coupled thermogravimetric and spectroscopic methods (TG/DSC/MS). Depending of the variant of raw nanotubes modification, the final products were oxygen- and nitrogen-doped materials of medium-developed surface area and mesoporous structure, showing highly diverse acidic-basic character of the surface, from the weakly acidic to slightly alkaline.
\end{abstract}

Keywords Carbon nanotubes - Thermogravimetry · Oxidation $\cdot$ Ammoxidation $\cdot$ Surface functional groups

The work was presented at Ninth International Symposium "Effects of Surface Heterogeneity in Adsorption and Catalysis on Solids", 17th-23rd July 2015, Wroclaw, Poland.

Małgorzata Gil

malgorzata.gil@poczta.umcs.lublin.pl

1 Laboratory of Applied Chemistry, Faculty of Chemistry, Adam Mickiewicz University in Poznań, Umultowska 89b, 61-614 Poznań, Poland

2 Laboratory of Optical Fibers Technology, Faculty of Chemistry, Maria Curie-Sklodowska University, Maria Curie-Sklodowska Sq. 5, 20-031 Lublin, Poland

\section{Introduction}

Carbonaceous materials play an important role not only in a purification of exhausted gases and waste water, but also as catalysts, catalyst supports or reducing agents (MarotoValer et al. 2005; Nowicki et al. 2012; Fahim et al. 2006; Kadirvelu et al. 2001; Bashkova et al. 2007; Muniz et al. 2000; Goscianska et al. 2012; Valente et al. 2003). Observed in recent years growth in the use of such materials is mainly caused by the relatively low cost of their production, rich resource base, as well as favorable physicochemical properties, such as highly developed surface area, high mechanical strength and chemical resistance and ease of degradation of used material. The unique properties of carbonaceous materials are in large extent conditioned by the type of raw material used for their production, the conditions of their preparation as well as by the chemical properties of the surface associated with the number, type and mode of binding of heteroatoms. Presence of the latter in the structure of materials influences largely their acid-base, hydrophobic-hydrophilic, sorption, catalytic, electrochemical as well as thermal properties.

Particularly popular among carbonaceous materials are that containing nitrogen and oxygen functional groups in their structure. The primary method of the generating oxygen functional group is a treatment of carbonaceous materials with various gases, such as oxygen, steam, carbon dioxide or nitrogen oxides (Rodriguez-Reinoso et al. 1995; Figueiredo et al. 1999; Pradhan and Sandle 1999) as well as with liquids oxidants (mainly $\mathrm{HNO}_{3}, \mathrm{H}_{2} \mathrm{O}_{2}$, and $\left(\mathrm{NH}_{4}\right)_{2} \mathrm{~S}_{2} \mathrm{O}_{8}$ (MorenoCastilla et al. 2000; Pietrzak et al. 2009; Malaika and Kozłowski 2011). The nitrogen-enriched materials can be obtained in a three different ways. The most often applied is thermal treatment of carbonaceous material in the presence 
of nitrogen supplying agent such as ammonia, urea, melamine, etc. (Nowicki et al. 2009; Bagreev et al. 2004; Kazmierczak-Razna et al. 2015; Pietrzak et al. 2006; Bimer et al. 1998). The second method consists in pyrolysis and activation of plastics including nitrogen species in their structure (Grzyb et al. 2009; Laszlo et al. 2001; Hsiao et al. 2011), while the last variant of nitrogen-modified materials preparation is a deposition of amines or imines on the carbonaceous material surface (Plaza et al. 2007; Tamai et al. 2006; Yin et al. 2007).

Although the functionalization of carbonaceous materials has been reported in many papers, there is still much to be learnt in this field. Therefore, the main aim of this study was preparation and physicochemical characterization of new carbonaceous materials obtained by means of ammoxidation (simultaneous oxidation and nitrogenation at elevated temperature) and oxidation of commercially available multi-walled carbon nanotubes. The effect of the particular modifications on the elemental composition, textural parameters as well as on acid-base and thermal properties of material prepared has been tested.

\section{Experimental}

\subsection{Materials preparation}

The investigation presented in this work was carried out on the commercial multi-walled carbon nanotubes manufactured by Sigma-Aldrich ${ }^{\circledR}$ (O.D 6-9 nm, L $5 \mu \mathrm{m}$, fixed carbon $>95 \%)$. The starting nanotubes (NT) were subjected to three different treatments: (1) ammoxidation (NTA sample), (2) oxidation with $20 \% \mathrm{HNO}_{3}$ (NTO sample) and (3) oxidation followed by ammoxidation (NTOA sample).

Ammoxidation (A) was performed with the mixture of ammonia and air at the ratio 1:3 (flow ratio of $\mathrm{NH}_{3}$ to air $250: 750 \mathrm{ml} / \mathrm{min}$ ) at the temperature of $350{ }^{\circ} \mathrm{C}$, for $5 \mathrm{~h}$.

Oxidation was performed according to the following procedure: $15 \mathrm{~g}$ of carbon nanotubes were placed in threenecked flask, equipped with a reflux condenser, dropping funnel and thermometer. Then, $100 \mathrm{ml}$ of $20 \% \mathrm{HNO}_{3}$ was added and the reaction mixture was heated up to boiling point. After that, the remaining portion of nitric acid (200 ml) was slowly added to the flask. After addition of the last portion of $\mathrm{HNO}_{3}$, the reaction was continued for $3 \mathrm{~h}$. The final product was recovered by filtration, washed repeatedly with hot distilled water and dried at $110{ }^{\circ} \mathrm{C}$.

\subsection{Analytical procedures}

The elemental analysis of the starting nanotubes and products obtained at each stage of the processing was performed on an elemental analyzer CHNS Vario EL III (Elementar Analysensysteme GmbH, Germany). The ash content was determined according to the ISO 1171:2002 standard: the dried sample was burned in a microwave oven at $850{ }^{\circ} \mathrm{C}$ for $60 \mathrm{~min}$.

The surface functional groups were determined according to Boehm method: $0.25 \mathrm{~g}$ of sample was placed in $25 \mathrm{ml}$ of the $0.1 \mathrm{M}$ sodium hydroxide and hydrochloric acid solutions. The vials were sealed and shaken for $24 \mathrm{~h}$, and then $10 \mathrm{ml}$ of each filtrate was pipetted and the excess of base or acid was titrated with $\mathrm{HCl}$ or $\mathrm{NaOH}$, respectively. The numbers of surface basic/acidic sites were calculated from the amount of hydrochloric acid/sodium hydroxide, which reacted with the sample.

The $\mathrm{pH}$ of the carbon nanotubes suspension, which can provides some information about the average acidity/basicity, was measured in the following way: a portion of $0.2 \mathrm{~g}$ the sample of nanotubes was added to $10 \mathrm{ml}$ of distilled water and the suspension was stirred overnight to reach equilibrium. Then the $\mathrm{pH}$ of the suspension was measured on a pHmeter manufactured by Metrohm Ion Analysis (Switzerland) equipped in Unitrode Pt1000 (combined glass $\mathrm{pH}$ electrode with temperature sensor).

Characterization of the pore structure of the samples was performed on the ground of low-temperature nitrogen adsorption/desorption isotherms measured on Autosorb iQ surface area analyzer, manufactured by Quantachrome Instruments (USA). Before the isotherm measurements, samples were outgassed at $150{ }^{\circ} \mathrm{C}$ for $8 \mathrm{~h}$. Surface area $\left(S_{\mathrm{BET}}\right)$ was calculated by Brunauer-Emmett-Teller method. The total pore volume $\left(V_{t}\right)$ was calculated by measuring the amount of liquid nitrogen adsorbed at a relative pressure $p / p_{0}=0.99$. Average pore diameter from equation $d=4 V_{t} / S_{\mathrm{BET}}$.

Thermal analysis was carried out on a STA 449 Jupiter F1, Netzsch (Selb, Germany) under the following operational conditions: heating rate $10{ }^{\circ} \mathrm{C} \min ^{-1}$, a dynamic atmosphere of helium $\left(50 \mathrm{ml} \mathrm{min}^{-1}\right)$ in the temperature range of $20-1000{ }^{\circ} \mathrm{C}$, sample mass of about $5 \mathrm{mg}$, sensor thermocouple type S TG-DSC. As a reference, empty $\mathrm{Al}_{2} \mathrm{O}_{3}$ crucible was used. The identification of gas composition coming out during decomposition process were detected and analysed by quadrupole mass spectrometer QMS 403C Aëolos (Germany) coupling online to STA instrument. The mass spectrometer was connected on-line to STA instrument by quartz capillary heated to $300{ }^{\circ} \mathrm{C}$. The QMS was operated with an electron impact ionizer with energy $70 \mathrm{eV}$. The measurements performed in scan mode for $m / z$, where $m$ is the mass of molecule and $z$ is a charge of the molecule in electron charge units in the range from 10 to $100 \mathrm{amu}$ allowed to identify all possible volatile particles produced during the decomposition. 


\section{Results and discussion}

\subsection{Elemental composition of materials studied}

The three variants of thermo-chemical treatment applied to nanotubes resulted in significant changes in the contribution of particular elements in their structure. As follows from the data collected in Table 1, each of the modifications performed caused a considerable decrease in the carbon content and simultaneously increase in the hydrogen, nitrogen and oxygen contribution. As expected, oxidation of the starting nanotubes leads mainly to introduction of significant amounts of oxygen into their structure. The small increase in the nitrogen content is a consequence of the fact, that due to the presence of aromatic rings (as a result of the reaction with $\mathrm{HNO}_{3}$ ), the carbon matrix was not only oxidized but also nitrated. In turn, exposure of the starting (NT) as well as pre-oxidized (NTO) nanotubes to ammonia-air mixture results in the introduction of significant amount of nitrogen in their structures and also significant changes in the contents of the other elements. However, the amount of nitrogen introduced into carbon nanotubes structure throughout ammoxidation process is significantly lower compared to activated carbon modified in the same manner (Nowicki et al. 2009). This fact confirms that structure of multiwalled carbon nanotubes is less susceptible to modification than that of activated carbons, so the conditions used for their ammoxidation (temperature, duration) should be more stringent.

Noteworthy is also different nature of the changes of oxygen content in case of NTA and NTOA samples. The decrease in the content of $\mathrm{O}^{\mathrm{daf}}$ observed for NTOA sample can be a consequence of the fact that part of oxygen functional groups was engaged in oxygen-nitrogen connections formation, whereas the increase in $\mathrm{O}^{\text {daf }}$ contribution observed for NTA sample results from the fact, that ammoxidation is process of simultaneous oxidation and nitrogenation of carbonaceous structure.

Table 1 Elemental composition of the raw and modified nanotubes (wt $\%$ )

\begin{tabular}{llllll}
\hline Sample & Ash & $\mathrm{C}^{\text {daf* }}$ & $\mathrm{H}^{\text {daf }}$ & $\mathrm{N}^{\text {daf }}$ & $\mathrm{O}^{\text {daf** }}$ \\
\hline NT & 0.2 & 98.0 & 0.1 & 0.1 & 1.8 \\
NTA & 0.9 & 91.5 & 1.1 & 3.4 & 4.0 \\
NTO & 1.1 & 93.2 & 0.3 & 0.4 & 6.1 \\
NTOA & 1.3 & 89.7 & 1.7 & 3.8 & 4.8 \\
\hline
\end{tabular}

* Dry-ash-free

** From difference

\subsection{Textural parameters of raw and modified nanotubes}

The results presented in Table 2 indicate that the starting multi-walled carbon nanotubes show not very well developed surface area $\left(271 \mathrm{~m}^{2} / \mathrm{g}\right)$ and remarkably mesoporous structure.

Each of the applied modifications brings about significant changes in their textural parameters. The decrease in the surface area is most pronounced for sample NTOA subjected to oxidation followed by ammoxidation, but for the remaining two samples the scale of changes is only slightly less. Modifications of nanotubes lead also to a considerable decrease in the total pore volume as well as mean pore diameter, which are about twice smaller than that of the initial material. Such drastic changes of these parameters are most probably caused by blocking of considerable part of pores by the oxygen and nitrogen functional groups introduced upon individual modifications.

\subsection{Acid-base properties of raw and modified nanotubes}

As follows from the data presented in Table 3, all the materials under investigation differ significantly in the number of acid and basic functional groups as well as in the surface $\mathrm{pH}$. The modifications of starting nanotubes (NT) lead to significant changes in the $\mathrm{pH}$, from weakly basic for NTA sample to weakly acidic in case of NTO sample, which is related to the presence of considerable amounts of basic or acidic functional groups generated in a consequence of exposure to ammonia-air mixture and nitric acid, respectively. In turn, sample NTOA, which was oxidized and next subjected to ammoxidation process, shows an intermediate acid-base character and its $\mathrm{pH}$ is slightly above 7 . The variant of nanotubes modification affects also the amount of generated functional groups. The highest total content of surface functional groups (among modified samples), reaching $1.01 \mathrm{mmol} / \mathrm{g}$ was found for sample NTO subjected only to oxidation process, while the lowest, of $0.57 \mathrm{mmol} / \mathrm{g}$, for NTA sample subjected only to ammoxidation. The character of functional groups present on the surface of particular materials also changes depending on the way of nanotubes modification. For sample NTOA and particularly NTO, the prevalence of acidic groups is observed, while in case of NTA sample the opposite tendency takes place.

\subsection{Thermal and spectroscopic studies}

The modified nanotubes were also subjected to thermal and spectroscopic analysis. By using the coupled methods (TG/ DSC/MS) it was possible to obtain complete 
Table 2 Textural parameters of the raw and modified nanotubes

\begin{tabular}{llll}
\hline Sample & Surface area $\left(\mathrm{m}^{2} / \mathrm{g}\right)$ & Total pore volume $\left(\mathrm{cm}^{3} / \mathrm{g}\right)$ & Average pore diameter $(\mathrm{nm})$ \\
\hline NT & 271 & 3.27 & 48.3 \\
NTA & 232 & 1.67 & 32.1 \\
NTO & 229 & 1.64 & 23.7 \\
NTOA & 211 & 1.57 & 25.4 \\
\hline
\end{tabular}

Table 3 Acid-base properties of the raw and modified nanotubes

\begin{tabular}{lllll}
\hline Sample & $\mathrm{pH}$ & Acidic groups $(\mathrm{mmol} / \mathrm{g})$ & Basic groups $(\mathrm{mmol} / \mathrm{g})$ & Total content $(\mathrm{mmol} / \mathrm{g})$ \\
\hline NT & 6.7 & 0.12 & 0.06 & 0.18 \\
NTA & 8.4 & 0.18 & 0.39 & 0.57 \\
NTO & 5.8 & 0.86 & 0.15 & 1.01 \\
NTOA & 7.2 & 0.54 & 0.42 & 0.96 \\
\hline
\end{tabular}

characterization of phenomena occurring in studied samples during the temperature increase simultaneously. Based on thermal analysis (TG) we get information about the temperature at which mass change occurs and about the quantity of this change. It is also possible to conclude thermal stability of the analysed samples. The knowledge what we get from differential scanning calorimetry (DSC) concerns exo- and endothermic phenomenon arising during the analysis (Menczel and Prime 2008). Identification of gas evolved during the temperature increase by quadrupole mass spectrometer (QMS) allows to predict probable mechanism of nanotubes degradation (Zielinski and Rajca 2000).

Based on thermoanalysis (Fig. 1) it was found that starting nanotubes (NT) have the highest thermal stability. Till $470{ }^{\circ} \mathrm{C}$ registered mass loss for this sample is only 0.7 $\mathrm{wt} \%$, and this loss should be ascribed as the result of water evaporation (physically adsorbed and water from the decomposition of functional oxygen group with lower thermal resistance, such as carboxyl groups).

Moreover, based on the calorimetric curve (Fig. 2) endothermic reaction can be observed (water evaporation), which is further confirmed by mass spectroscopy analysis (Fig. 3). Based on which we can see a change in the amount of ion $\mathrm{m}$ : 18 responsible for the presence of water in the analyzed gases. This ion is present to a temperature of $120{ }^{\circ} \mathrm{C}$, after which its content is increasing again at $230{ }^{\circ} \mathrm{C}$ and continues to increase until the end of analysis. In the same temperature ranges, but with a much lower intensity, ion $\mathrm{m}: 17$ which indicates the presence of a hydroxyl group is present. For unmodified nanotubes proper degradation starts at $605{ }^{\circ} \mathrm{C}$ and at $1000{ }^{\circ} \mathrm{C}$ a $5.7 \%$ wt $\%$ of mass loss is observed. Based on the results obtained from mass spectroscopy (Fig. 3) a slight decrease in the
Fig. 1 Mass loss for all tested nanotubes

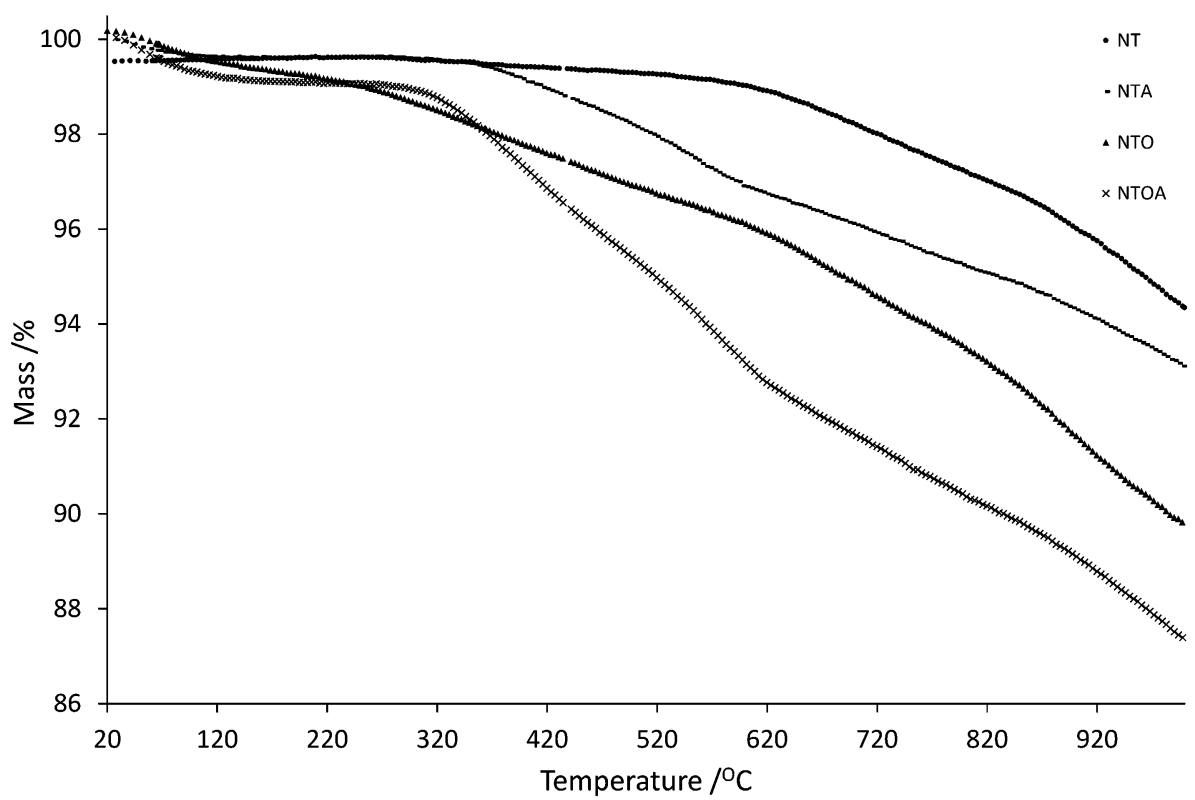


Fig. 2 Calorimetric curves for all tested nanotubes
Fig. 3 Temperature distribution for ions of unmodified nanotubes
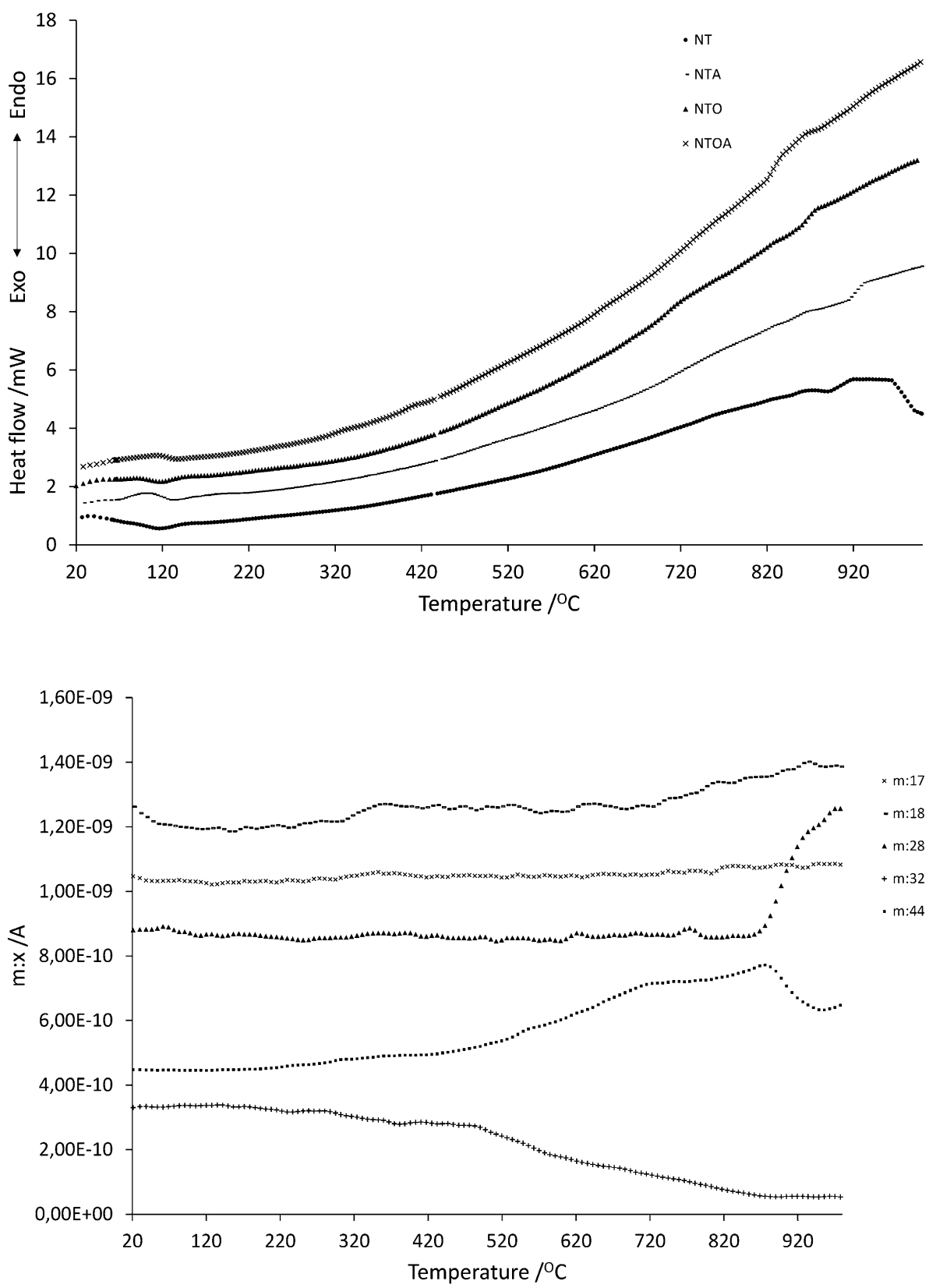

content of ion $\mathrm{m}: 28$, (characteristic for carbon monoxide) at the temperature $70{ }^{\circ} \mathrm{C}$ up to the temperature of $275^{\circ} \mathrm{C}$ is observed. At the same temperature range slight decrease in the content of ion m: 32 (characteristic for oxygen) and increase in the content of ion m: 44 (characteristic for carbon dioxide) is observed. Most probably, present in the reaction chamber carbon monoxide is oxidized to carbon dioxide. Our finding additionally confirms mass change at this temperature range, which is only $0.02 \mathrm{wt} \%$. From $275^{\circ} \mathrm{C}$ a significant change in the content of ions $\mathrm{m}: 32$ and 44, with constant content of ion m: 28 can be observed. These changes indicate oxidation of carbon to carbon dioxide. At a temperature of $850{ }^{\circ} \mathrm{C}$ a sudden drop in the carbon dioxide content while significantly increase in the content of carbon monoxide occurs. Most likely insufficient oxygen content contributes to the oxidation of carbon only to carbon monoxide. At this point, it must be emphasized that the analysis is carried out under inert atmosphere but the oxygen present in the reaction chamber cannot be eliminate.

The ammoxidised nanotubes (NTA) shows a slightly lower thermal resistance compared to unmodified nanotubes. Till the temperature of $1000{ }^{\circ} \mathrm{C} 7 \mathrm{wt} \%$ of mass loss is registered. The initial mass loss $\left(0.4 \mathrm{wt} \%\right.$ till $\left.160{ }^{\circ} \mathrm{C}\right)$ is the result of water evaporation, which confirms both present on the calorimetric curve (Fig. 2) an endothermic 
process and the change of ion m: 18 content on the mass spectroscopy graph (Fig. 4). From $160{ }^{\circ} \mathrm{C}$ the content of ion $\mathrm{m}: 18$ increases and keep increasing till the end of the analysis. Ion m: 17 may be responsible for the presence of ammonia, which taking into account the ammoxidation, is possible. Unfortunately, the presence of ion $\mathrm{m}$ : 17 in the same temperature ranges as the water indicates that it comes from a hydroxyl group. Only at the temperature range $510-620^{\circ} \mathrm{C}$ an increase in the intensity of this ion, with a simultaneous decrease of ion content characteristic for the water is observed, which may indicate the presence of ammonia. In the same range ion m: 16 appears, which indicates the presence of amino group. Both ions indicate the basic nature of the surface groups. In the discussed sample, similarly as it was in the case of the unmodified sample, a slight decrease of ion m: 28 in the temperature range $65-283{ }^{\circ} \mathrm{C}$, while a small loss of ion m: 32 and increase of ion m: 44 based on mass spectroscopy analysis (Fig. 4) was noted.

At the temperature of $283{ }^{\circ} \mathrm{C}$ an increase in the content of ions m: 28 and 44 can be observed. Apart from the obvious statement that they indicate presence of carbon monoxide and carbon dioxide, the possibility that they can also come from other substances cannot be eliminate. Which further confirms their decidedly different shape when compared with the unmodified sample. Given that the nanotubes were exposure to ammonia-air mixture, we came to the conclusion that ion $\mathrm{m}$ : 28 comes from a quinone group, which confirms the increase of amount of acidic functional groups (Table 3). In contrast, ion m: 44 may correspond to either the presence of secondary amines and amide groups, which confirms the increase of amount of basic functional groups (Table 3). From $840{ }^{\circ} \mathrm{C}$ a significant decrease in the content of ion m: 44 and increase of ion m: 28 occurs. It indicates the oxidation process of carbon to carbon monoxide. At the temperature range 283-840 ${ }^{\circ} \mathrm{C}$ appears ion m: 30, which indicates the presence of the nitroso group.

The mass loss for nanotubes subjected to oxidation with $20 \% \mathrm{HNO}_{3}$ (NTO) recorded at $1000{ }^{\circ} \mathrm{C}$ was 10.2 wt $\%$. As in all analysed samples, the mass loss at the beginning is caused by water evaporation. Based on mass spectroscopy studies (Fig. 5), it can be concluded that this process continues until a temperature of about $190{ }^{\circ} \mathrm{C}$, which is confirmed by the endothermic reaction. Until this temperature ion $\mathrm{m}: 17$ is also present. From $190{ }^{\circ} \mathrm{C}$ continuous growth in the content of ion m: 18 can be observed. Looking at the TG curve (Fig. 1) it can be seen that for all analysed samples, two distinct stages of mass loss can be observed. The first relates to the mass loss due to water evaporation, and then plateau can be observed wherein the sample practically do not lose mass, and then it comes to the proper degradation. For sample NTO from the beginning of the process mass loss is observed.

At $110{ }^{\circ} \mathrm{C}$ a significant change in the content of ions $\mathrm{m}$ : 32 and 44, with constant content of ion m: 28 can be observed. These changes indicate oxidation of carbon to carbon dioxide. However it should be noted that the curve shapes of the ion m: 28 and 44 are different compared to those present in the unmodified nanotubes. This variation relates to the temperature range of $600-720^{\circ} \mathrm{C}$, which probably indicates the possibility presence of both quinone groups (m: 28) amide and secondary amine groups (m: 44). Which is confirmed by the increase in the amount of basic and acidic functional groups (Table 3 ).
Fig. 4 Temperature distribution for ions of ammoxidised nanotubes

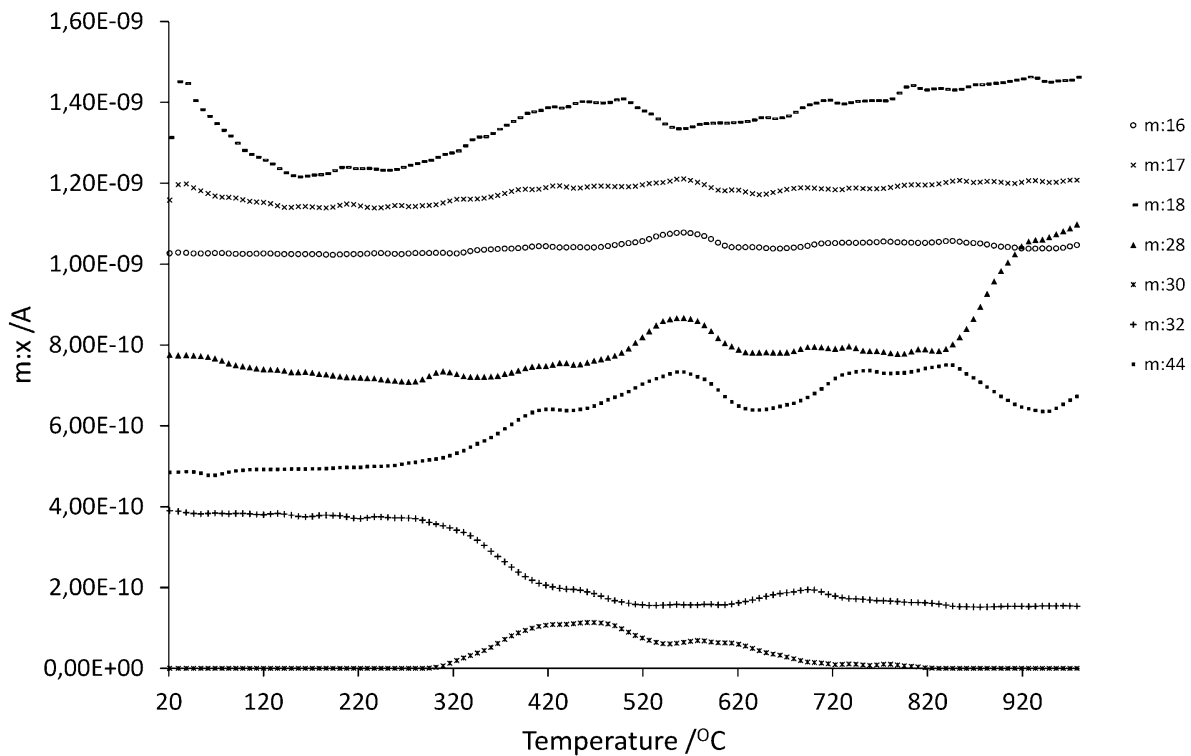


Fig. 5 Temperature distribution for ions of oxidised nanotubes

Fig. 6 Temperature distribution for ions of oxidised and then ammoxidised nanotubes
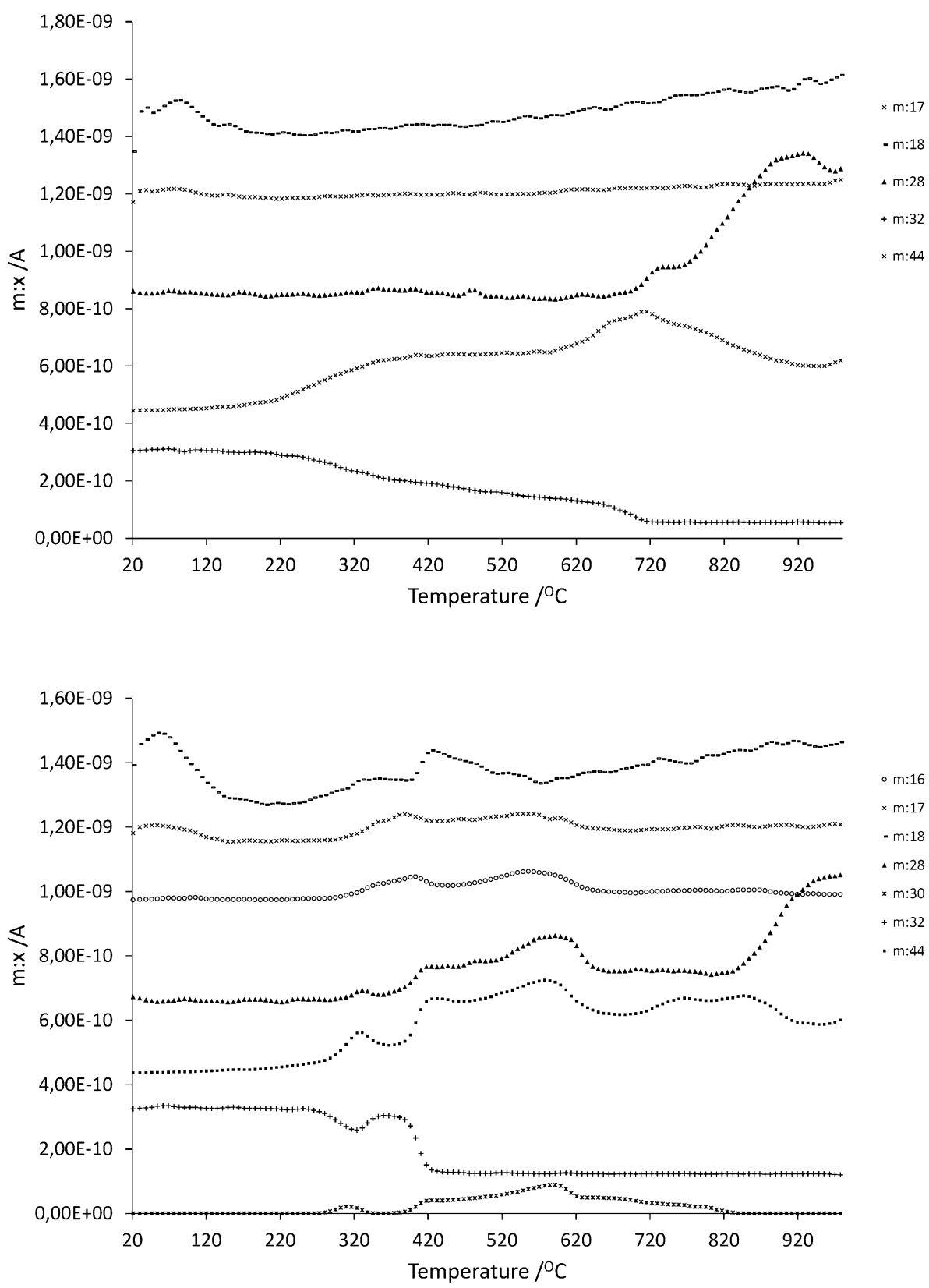

At a temperature of $720{ }^{\circ} \mathrm{C}$ a decrease in the content of ion m: 44 and increase of ion m: 28 occurs. It indicates the oxidation process of carbon to carbon monoxide.

The oxidised and then ammoxidised nanotubes (NTOA) have the lowest thermal stability. To a temperature of $1000{ }^{\circ} \mathrm{C}$ mass loss of $12.6 \% \mathrm{wt} \%$ is observed. Spectroscopic characterization indicates a very strong similarity between the sample NTA and NTOA. Exposing the sample to oxidation and in the next step to ammoxidation not substantially affect the thermal and spectroscopy characteristic of the nanotubes (Fig. 6).

\section{Conclusion}

The results obtained have shown, that by ammoxidation and oxidation of commercial nanotubes it possible to prepare in a simple way a wide spectrum of new carbonaceous materials, showing a completely different elemental composition, textural parameters as well as acid-base properties of the surface in comparison to the raw materials. The character of changes induced significantly depends on the type of the thermo-chemical treatment and are most pronounced for nanotubes subjected to oxidation followed by 
ammoxidation. Based on the results obtained from mass spectroscopy the presence of amino group, secondary amines and amide groups in the structure of the nanotubes subjected to ammoxidation has been found. In contrast, sample subjected to oxidation is characterized mainly by the presence of quinone groups. Moreover, the thermal analysis has proved that the functional groups introduced into the carbon nanotubes structure in a very high extent affect their thermal stability.

Acknowledgments This work was partially supported by Ministry of Science and Higher Education of Poland (Project Iuventus Plus No. IP2012 004072).

Open Access This article is distributed under the terms of the Creative Commons Attribution 4.0 International License (http://crea tivecommons.org/licenses/by/4.0/), which permits unrestricted use, distribution, and reproduction in any medium, provided you give appropriate credit to the original author(s) and the source, provide a link to the Creative Commons license, and indicate if changes were made.

\section{References}

Bagreev, A., Angel Menendez, J., Dukhno, I., Tarasenko, Y., Bandzosz, T.J.: Bituminous coal-based activated carbons modified with nitrogen as adsorbents of hydrogen sulfide. Carbon 42(3), 469-476 (2004)

Bashkova, S., Baker, F.S., Wu, X., Armstrong, T.R., Schwartz, V.: Activated carbon catalyst for selective oxidation of hydrogen sulphide: on the influence of pore structure, surface characteristics, and catalytically-active nitrogen. Carbon 45, 1354-1363 (2007)

Bimer, J., Sałbut, P.D., Berłożecki, S., Boudou, J.P., Broniek, E., Siemieniewska, T.: Modified active carbons from precursors enriched with nitrogen functions: sulfur removal capabilities. Fuel 77, 519-525 (1998)

Fahim, N.F., Barsoum, B.N., Eid, A.E., Khalil, M.S.: Removal of chromium (III) from tannery wastewater using activated carbon from sugar industrial waste. J. Hazard. Mater. 136(2), 303-309 (2006)

Figueiredo, J.L., Pereira, M.F.R., Freitas, M.M.A., Orfao, J.J.M.: Modification of the surface chemistry of activated carbons. Carbon 37, 1379-1389 (1999)

Goscianska, J., Nowak, I., Nowicki, P., Pietrzak, R.: The influence of silver on the physicochemical and catalytic properties of activated carbons. Chem. Eng. J. 189-190, 422-430 (2012)

Grzyb, B., Albiniak, A., Broniek, E., Furdin, G., Mareche, J.F., Begin, D.: $\mathrm{SO}_{2}$ adsorptive properties of activated carbons prepared from polyacrylonitrile and its blends with coal-tar pitch. Microporous Mesoporous Mater. 118, 163-168 (2009)

Hsiao, H.-Y., Huang, C.-M., Hsu, M.-Y., Chen, H.: Preparation of high-surface-area PAN-based activated carbon by solutionblowing process for $\mathrm{CO}_{2}$ adsorption. Sep. Purif. Technol. 82, 19-27 (2011)
Kadirvelu, K., Thamaraselvi, K., Namasivayam, C.: Removal of heavy metals from industrial wastewaters by adsorption onto activated carbon prepared from an agricultural solid waste. Bioresour. Technol. 76(1), 63-65 (2001)

Kazmierczak-Razna, J., Nowicki, P., Pietrzak, R.: The use of microwave radiation for obtaining activated carbons enriched in nitrogen. Powder Technol. 273, 71-75 (2015)

Laszlo, K., Tombacz, E., Josepovits, K.: Effect of activation on the surface chemistry of carbons from polymer precursors. Carbon 39, 1217-1228 (2001)

Maroto-Valer, M.M., Tang, Z., Zhang, Y.: $\mathrm{CO}_{2}$ capture by activated and impregnated anthracites. Fuel Process. Technol. 86, 1487-1502 (2005)

Menczel, J.D., Prime, R.B.: Thermal analysis of polymers. John Wiley \& Sons, Hoboken (2008)

Moreno-Castilla, C., Lopez-Ramon, M.V., Carrasco-Marin, F.: Changes in surface chemistry of activated carbons by wet oxidation. Carbon 38, 1995-2001 (2000)

Muniz, J., Marban, G., Fuertes, A.B.: Low temperature selective catalytic reduction of no over modified activated carbon fibres. Appl. Catal. B Environ. 27, 27-36 (2000)

Nowicki, P., Pietrzak, R., Wachowska, H.: Influence of the precursor metamorphism degree on preparation of nitrogen enriched activated carbons by ammoxidation and chemical activation of coals. Energy Fuels 23, 2205-2212 (2009)

Nowicki, P., Supłat, M., Przepiórski, J., Pietrzak, R.: $\mathrm{NO}_{2}$ removal on adsorbents obtained by pyrolysis and physical activation of corrugated cardboard. Chem. Eng. J. 195-196, 7-14 (2012)

Pietrzak, R., Wachowska, H., Nowicki, P.: Preparation of nitrogenenriched activated carbons from brown coal. Energy Fuel. 20, 1275-1280 (2006)

Pietrzak, R., Nowicki, P., Wachowska, H.: The influence of oxidation with nitric acid on the preparation and properties of active carbon enriched in nitrogen. Appl. Surf. Sci. 255, 3586-3593 (2009)

Plaza, M.G., Pevida, C., Arenillas, A., Rubiera, F., Pis, J.J.: $\mathrm{CO}_{2}$ capture by adsorption with nitrogen enriched carbons. Fuel $\mathbf{8 6}$, 2204-2212 (2007)

Pradhan, B.K., Sandle, N.K.: Effect of different oxidizing agent treatments on the surface properties of activated carbons. Carbon 37, 1323-1332 (1999)

Rodriguez-Reinoso, F., Molina-Sabio, M., Gonzalez, M.T.: The use of steam and $\mathrm{CO}_{2}$ as activating agents in the preparation of activated carbons. Carbon 33, 15-23 (1995)

Tamai, H., Shiraki, K., Shiono, T., Yasuda, H.: Surface functionalization of mesoporous and microporous activated carbons by immobilization of diamine. J. Colloid Interface Sci. 295, 299-302 (2006)

Valente, A., Palma, C., Fonseca, I.M., Ramos, A.M., Vital, J. Oxidation of pinane over phthalocyanine complexes supported on activated carbon: Effect of the support surface treatment. Carbon 41, 2793-2803 (2003)

Yin, C.Y., Aroua, M.K., Wandaud, W.M.A.: Impregnation of palm shell activated carbon with polyethyleneimine and its effects on $\mathrm{Cd}^{2+}$ adsorption. Colloids Surf. A 307, 128-136 (2007)

Zielinski, W., Rajca, A.: Metody spektroskopowe i ich zastosowanie do identyfikacji związków organicznych. WNT, Warszawa (2000) 